\title{
Effect of Static Disorder in an Electron Fabry-Perot Interferometer with Two Quantum Scattering Centers
}

\author{
Francesco Ciccarello, 2, and G. Massimo Palma ${ }^{2}$, Michelangelo Zarcone ${ }^{1}$, Yasser Omar ${ }^{3}$, Vitor Rocha Vieira ${ }^{4}$ \\ ${ }^{1}$ CNISM and Dipartimento di Fisica e Tecnologie Relative dell'Università degli Studi di Palermo, \\ Viale delle Scienze, Edificio 18, I-90128 Palermo, Italy \\ ${ }^{2}$ NEST-INFM (CNR) \& Dipartimento di Scienze Fisiche ed Astronomiche \\ dell'Università degli Studi di Palermo, Via Archirafi 36, I-90123 Palermo, Italy \\ 3 SQIG, Instituto de Telecomunicações, P-1049-001 Lisbon and CEMAPRE, \\ ISEG, Technical University of Lisbon, P-1200-781 Lisbon, Portugal \\ ${ }^{4}$ Department of Physics, Instituto Superior Técnico, Av. Rovisco Pais, 1049-001 Lisbon, Portugal
}

\begin{abstract}
In a recent paper - F. Ciccarello et al., New J. Phys. 8, 214 (2006) - we have demonstrated that the electron transmission properties of a one-dimensional (1D) wire with two identical embedded spin-1/2 impurities can be significantly affected by entanglement between the spins of the scattering centers. Such effect is of particular interest in the control of transmission of quantum information in nanostructures and can be used as a detection scheme of maximally entangled states of two localized spins. In this letter, we relax the constraint that the two magnetic impurities are equal and investigate how the main results presented in the above paper are affected by a static disorder in the exchange coupling constants of the impurities. Good robustness against deviation from impurity symmetry is found for both the entanglement dependent transmission and the maximally entangled states generation scheme.
\end{abstract}

PACS numbers: 03.67.Mn, 72.10.-d, 73.23.-b, 85.35.Ds

The key role that entanglement plays in quantum information processing has been investigated over the past few years [1]. In this framework, the role that it plays in quantum transport in mesoscopic systems has been analyzed [2]. Recently, we have shown a novel way in which entanglement can be used for controlling electron transport in nanostructures [3]. Assume to have a 1D wire where two spin-1/2 impurities are embedded at a fixed distance. Such system can be regarded as the electron analogue of a Fabry-Perot (FP) interferometer, with the impurities playing the role of two mirrors with a spin quantum degree of freedom. Single electrons are injected into the wire and undergo multiple scattering between the two magnetic impurities due to the presence of a contact exchange electron-impurity coupling. At each scattering event spin-flip may occur and thus the transmitted spin state of the overall system will be generally different from the incoming one. The typical behaviour shown by electron transmittivity $T$ consists of a loss of electron coherence and thus of a resonance condition $T=1$, due to the presence of internal spin degrees of freedom of the scattering centers [4]. Such a system is indeed the electron analogue of a Fabry-Perot (FP) interferometer, with the impurities playing the role of two mirrors with a spin quantum degree of freedom. However, unlike the standard FP device where scattering with each mirror introduces a well-fixed phase shift, in the present system the above phase shifts depend on the electron-impurities spin state and thus, in general, a resonance condition cannot take place. However, the presence of quantum scatterers allows one to investigate if and to what extent maximally entangled states of the impurity spins can affect electron transmission. Denot- ing by $\left|\Psi^{ \pm}\right\rangle=2^{-\frac{1}{2}}(|\uparrow \downarrow\rangle \pm|\downarrow \uparrow\rangle)$ the triplet and singlet maximally entangled spin states of the impurities, respectively, we have thus found that when $\left|\Psi^{-}\right\rangle$is prepared, a perfect resonance condition $T=1$ can be always reached at electron wave vectors fulfilling $k x_{0}=n \pi$ ( $n$ integer, $x_{0}$ the distance between the impurities) and regardless of the electron spin state. When this occurs the incoming spin state of the electron-impurities system is transmitted completely unchanged. Therefore, a sort of perfect "transparency" takes place [3]. Moreover, as illustrated in Fig. 1, electron transmission within the one spin-up family of impurity states $\cos \vartheta|\uparrow \downarrow\rangle+e^{i \varphi} \sin \vartheta|\downarrow \uparrow\rangle$ is maximized (minimized) by $\left|\Psi^{-}\right\rangle\left(\left|\Psi^{+}\right\rangle\right)$. $T$ is thus crucially affected by the relative phase $\varphi$. This suggests the appealing possibility to use entanglement between the impurity spins for controlling electron transmission in a 1D wire or, alternatively, for implementing a maximally entangled states detection scheme via electron transmission. The above phenomena have been demonstrated to follow from an effective conservation law occurring whenever the condition $k x_{0}=n \pi$ is fulfilled. In [3] the following Hamiltonian has been assumed

$$
H=\frac{p^{2}}{2 m^{*}}+J \boldsymbol{\sigma} \cdot \mathbf{S}_{1} \delta(x)+J \boldsymbol{\sigma} \cdot \mathbf{S}_{2} \delta\left(x-x_{0}\right)
$$

where $p=-i \hbar \nabla, m^{*}$ and $\boldsymbol{\sigma}$ are the electron momentum operator, effective mass and spin- $1 / 2$ operator respectively, $\mathbf{S}_{i}(i=1,2)$ is the spin- $1 / 2$ operator of the $i$-th impurity and $J$ is the exchange spin-spin coupling constant between the electron and each impurity. Denoting by $\mathbf{S}=\boldsymbol{\sigma}+\mathbf{S}_{1}+\mathbf{S}_{2}$ and $\mathbf{S}_{12}=\mathbf{S}_{1}+\mathbf{S}_{2}$ the total spin of the system and of the two impurities, respectively, Hamiltonian (1) implies conservation of $\mathbf{S}^{2}$ and $S_{z} \cdot \mathbf{S}_{12}^{2}$ 


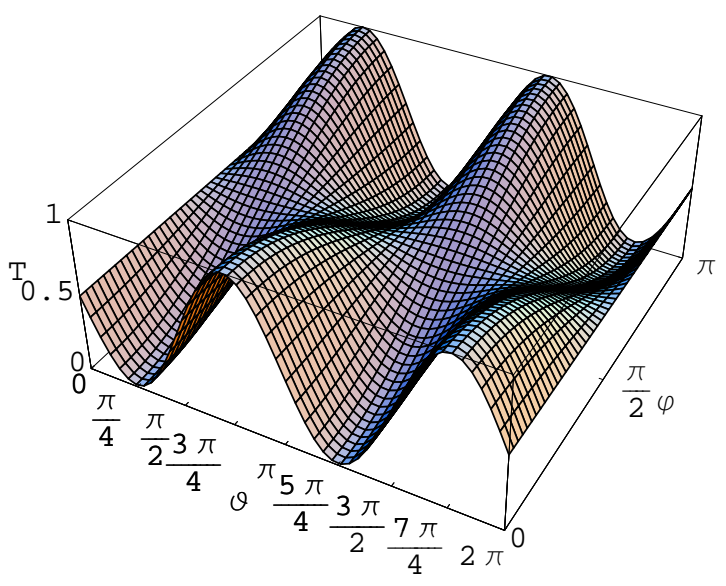

FIG. 1: Electron transmittivity $T$ at $k x_{0}=n \pi$ and $\rho(E) J=$ 10 when the electron is injected in an arbitrary spin state with the impurities prepared in the state $\cos \vartheta|\uparrow \downarrow\rangle+e^{i \varphi} \sin \vartheta|\downarrow \uparrow\rangle$. $\rho(E)$ is the density of states per unit length of the wire.

is in general not conserved due to the difference between $\delta(x)$ and $\delta\left(x-x_{0}\right)$ in (11). However, when $k x_{0}=n \pi$ the effective representations $\delta_{k}(x)$ and $\delta_{k}\left(x-x_{0}\right)$ of these two electron orbital operators coincide (the electron being found at $x=0$ and $x=x_{0}$ with equal probability) and $\mathbf{S}_{12}^{2}$ turns out to be an additional constant of motion. This fact is the ultimate reason for the occurrence of the above mentioned behaviours associated with $\left|\Psi^{-}\right\rangle$ and $\left|\Psi^{+}\right\rangle$(note that these are eigenstates of $\mathbf{S}_{12}^{2}$ ), as explained in detail in [3].

However, the above effective conservation law of $\mathbf{S}_{12}^{2}$ relies on the assumption of dealing with two perfectly identical impurities with equal coupling constant $J$. Of course, due to unavoidable static disorder, this condition cannot be strictly realized in a real system. The aim of this paper is to investigate how large the difference between the coupling constants of the two impurities can be before the entanglement dependent effects and the maximally entangled states generation scheme presented in [3] are significantly spoiled.

To begin with, let $J_{i}(i=1,2)$ be the exchange coupling constant of the $i$-th impurity. We thus generalize Hamiltonian (1) as

$$
H=\frac{p^{2}}{2 m^{*}}+J_{1} \boldsymbol{\sigma} \cdot \mathbf{S}_{1} \delta(x)+J_{2} \boldsymbol{\sigma} \cdot \mathbf{S}_{2} \delta\left(x-x_{0}\right)
$$

It is convenient to introduce the quantities $\bar{J}=\left(J_{1}+\right.$ $\left.J_{2}\right) / 2$ and $\Delta J=J_{2}-J_{1}$ through which $J_{i}(i=1,2)$ can be expressed as $J_{1}=\bar{J}-\Delta J / 2$ and $J_{2}=\bar{J}+\Delta J / 2$. Our previous results with identical impurities are thus recovered for $\Delta J \rightarrow 0$. The exact stationary states of the system at all orders in $J_{1}$ and $J_{2}$ can be derived through an appropriate quantum waveguide theory approach. Since $\mathbf{S}^{2}$ and $S_{z}$ are still constants of motion when $J_{1} \neq J_{2}$, the block diagonalization-based procedure used for the

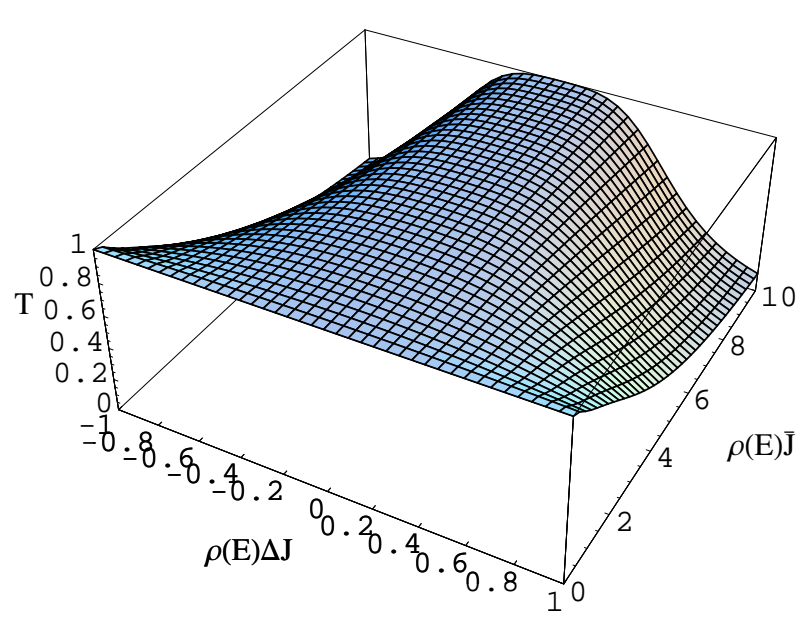

FIG. 2: $T$ versus $\rho(E) \bar{J}$ and $\rho(E) \Delta J$ for $k x_{0}=n \pi$ when the electron is injected in an arbitrary spin state with the impurities prepared in the state $\left|\Psi^{-}\right\rangle . \rho(E) \Delta J$ is normalized to $\rho(E) \bar{J}$, reducing to the ratio $\Delta J / \bar{J}$.

case of two identical impurities [3] can be readopted, the difference being that in the present case there is an additional parameter. Denoting the total spin of the electron and the $i$-th impurity as $\mathbf{S}_{e i}=\boldsymbol{\sigma}+\mathbf{S}_{i}$ and assuming leftincident electrons, we use as spin-space basis the states $\left|s_{e 2} ; s, m_{s}\right\rangle$, common eigenstates of $\mathbf{S}_{e 2}^{2}, \mathbf{S}^{2}$ and $S_{z}$, to express, for a fixed wave vector $k>0$, each of the eight stationary states of the system as an $8 \mathrm{D}$ column. The calculation of the stationary states through suitable boundary conditions [3] allows us to find all the transmission probability amplitudes $t_{s_{e 2} 2}^{\left(s_{e 2}^{\prime}, s\right)}$ that an electron prepared in the incoming state $|k\rangle\left|s_{e 2}^{\prime} ; s, m_{s}\right\rangle$ is transmitted in a state $|k\rangle\left|s_{e 2} ; s, m_{s}\right\rangle$. These coefficients can be used to compute how an electron is transmitted through the wire for any arbitrary initial spin state of the system [3]. The transmission amplitudes $t_{s_{e 2}}^{\left(s_{e 2}^{\prime}, s\right)}$ turn out to be functions of the three dimensionless parameters $k x_{0}, \rho(E) \bar{J}$ and $\rho(E) \Delta J$, where $\rho(E)=\left(\sqrt{2 m^{*} / E}\right) / \pi \hbar$ is the density of states per unit length of the wire as a function of the electron energy $E$.

We begin our analysis by investigating how the effect of perfect transparency shown when the impurity spins are prepared in the singlet state is affected by a difference in the two coupling constants. In Fig. 2 we plot the electron transmittivity $T$ versus $\rho(E) \bar{J}$ and $\rho(E) \Delta J$ when the electron is injected in an arbitrary spin state for $k x_{0}=n \pi$ with the impurities in the state $\left|\Psi^{-}\right\rangle$. Note that $T=1$ for $\Delta J=0$ since the case of perfect transparency with identical impurities is recovered. As expected, for a fixed $\rho(E) \bar{J}, T$ decreases for increasing values of $|\Delta J|$ due to the progressive lack of conservation of $\mathbf{S}_{12}^{2}$. Note how this decrease gets faster for increasing strengths of $\rho(E) \bar{J}$, indicating that for a given 
electron energy low coupling constants $\bar{J}$ show better robustness against impurities'asymmetry. It turns out that for a difference in the coupling constants larger than $25 \%$ compared to $\bar{J}$, perfect transparency is not significantly spoiled $(T>0.95)$ in the whole broad range of strengths of $\rho(E) \bar{J}$ here considered.

A remarkable feature of the plot in Fig. 2 is its symmetry with respect to a change of sign in $\Delta J$ for a fixed $\bar{J}$ (the relevant parameter being thus $|\Delta J|$ ). To explain this, we recall that, for $k x_{0}=n \pi, \delta_{k}(x)=\delta_{k}\left(x-x_{0}\right)$ and write the non-kinetic part $V$ of Hamiltonian (2) in the form

$$
V=\left[\bar{J} \boldsymbol{\sigma} \cdot\left(\mathbf{S}_{1}+\mathbf{S}_{2}\right)+\frac{\Delta J}{2} \boldsymbol{\sigma} \cdot\left(\mathbf{S}_{2}-\mathbf{S}_{1}\right)\right] \delta_{k}(x)
$$

Note that in such regime, where the electron has equal probability to be found at $x=0$ and at $x=x_{0}$, a change in the sign of $\Delta J$ is equivalent to an interchange of the impurity indexes. Therefore, the above symmetry property of $T$ in the case of Fig. 2 straightforwardly follows from the symmetry of $\left|\Psi^{-}\right\rangle$under an interchange of impurity 1 and 2 . In the remaining of this paper, we will thus consider only positive values of $\Delta J$ whenever the initial spin state is symmetric for an interchange of the two impurities, such in the cases of $\left|\Psi^{+}\right\rangle$and $|\downarrow \downarrow\rangle$.

Denoting by $T_{\Psi^{ \pm}}$the value of $T$ obtained for $\left|\Psi^{ \pm}\right\rangle$, it is worth analyzing the behaviour of $\Delta T=T_{\Psi^{-}}-T_{\Psi^{+}}$, that is the difference of the electron transmittivity for $\left|\Psi^{-}\right\rangle$ (high transmission) and $\left|\Psi^{+}\right\rangle$(low transmission). In order to observe the entanglement controlled-transmittivity and/or detect maximally entangled singlet/triplet states, one aims at having the highest possible value of $\Delta T[3]$ with the hope that it is only weakly affected by some impurities' asymmetry. Regarding the latter issue, in Fig. 3 we plot $\Delta T$ normalized to its value for $\Delta J=0$ versus $\rho(E) \bar{J}$ and $\rho(E) \Delta J$ in the same regime considered in Fig. 2. A behaviour qualitatively very similar to the case of Fig. 2 is exhibited. In the whole considered range of $\rho(E) \bar{J}, \Delta T$ turns out to be only weakly affected $(\Delta T>0.95)$ by a difference in the impurity coupling constants up to more than $25 \%$.

To observe the entanglement-dependent electron transmittivity one must of course be able to prepare the maximally entangled states $\left|\Psi^{-}\right\rangle$and $\left|\Psi^{+}\right\rangle$. These states can be easily transformed into each other by simply introducing a relative phase shift through a local field. In [3] we have proposed a scheme to generate the state $\left|\Psi^{+}\right\rangle$via electron scattering, improving a previous recent proposal [5]. The idea is to inject an electron in the state $|\uparrow\rangle$ in the regime $k x_{0}=n \pi$ with the two impurity spins initially in the state $|\downarrow \downarrow\rangle$. Due to conservation of both $\mathbf{S}_{12}^{2}$ and $S_{z}$, when the electron is transmitted in the state $|\downarrow\rangle$ with probability $T_{\downarrow}$, the two impurities are projected into the state $\left|\Psi^{+}\right\rangle[3]$. A difference in the coupling constants of the impurities is expected to modify the spin-polarized transmission probability $T_{\downarrow}$. Moreover, since the scheme

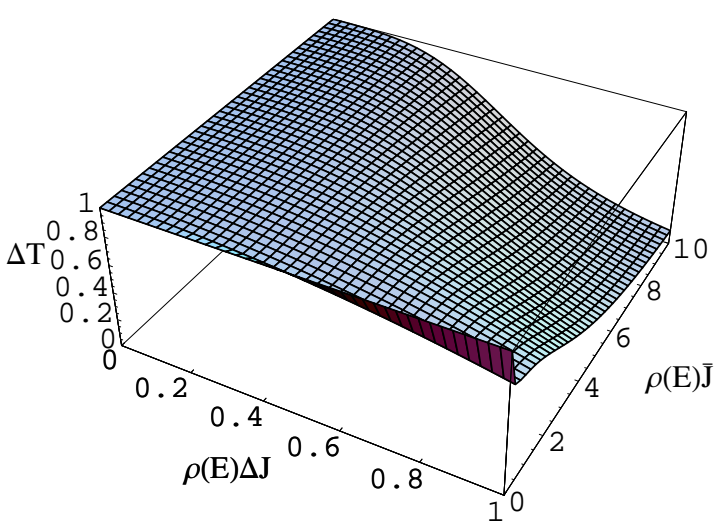

FIG. 3: $\Delta T=T_{\Psi^{-}}-T_{\Psi^{+}}$versus $\rho(E) \bar{J}$ and $\rho(E) \Delta J$ for $k x_{0}=n \pi$ when the electron is injected in an arbitrary spin state. $\rho(E) \Delta J$ is normalized to $\rho(E) \bar{J}$, reducing to the ratio $\Delta J / \bar{J} . \Delta T$ is normalized to its value for $\Delta J=0$.

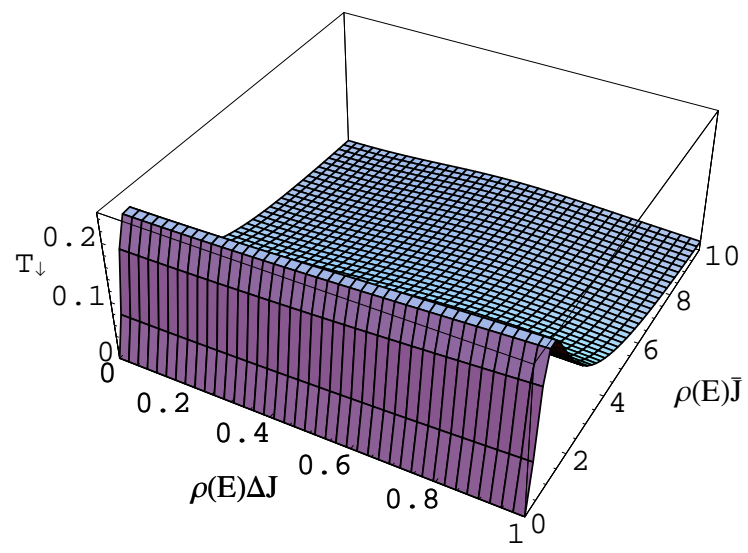

FIG. 4: $T_{\downarrow}$ versus $\rho(E) \bar{J}$ and $\rho(E) \Delta J$ for $k x_{0}=n \pi$ when the electron is injected in the state $|\uparrow\rangle$ with the impurities prepared in the state $|\downarrow \downarrow\rangle . \rho(E) \Delta J$ is normalized to $\rho(E) \bar{J}$, reducing to the ratio $\Delta J / \bar{J}$.

relies on the conservation of $\mathbf{S}_{12}^{2}$, the two localized spins in general will not be projected in the maximally entangled state $\left|\Psi^{+}\right\rangle$. In Figs. 4 and 5 we thus plot respectively $T_{\downarrow}$ and the fidelity $F_{\Psi^{+}}$with respect to $\left|\Psi^{+}\right\rangle$of the (normalized) spin state into which the impurities are projected after the electron is transmitted in the spin down state. $T_{\downarrow}$ is almost negligibly affected by the presence of $\Delta J$. The same is not true for $F_{\Psi^{+}}$which is indeed expected to be very sensible to the lack of conservation of $\mathbf{S}_{12}^{2} . \quad F_{\Psi^{+}}>0.95$ in the whole considered range of $\rho(E) \bar{J}$ for a difference in the impurity coupling constants up to more than $5 \%$. However, for $\rho(E) \bar{J} \simeq 1$, that is the strength of the impurity coupling constant maximizing $T_{\downarrow}, F_{\Psi^{+}}>0.95$ up to values of $|\Delta J| / \bar{J}$ larger than $30 \%$. 


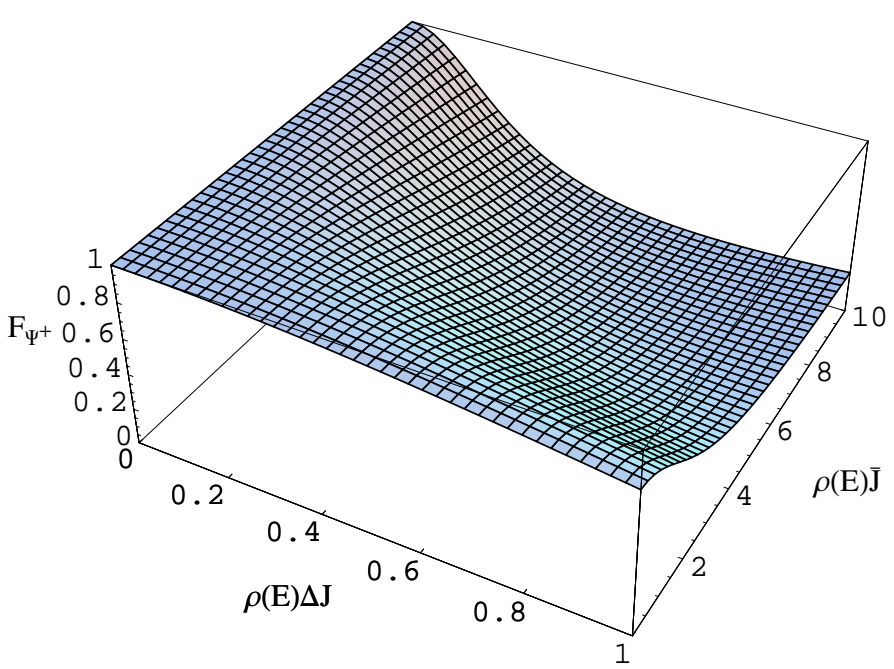

FIG. 5: $F_{\Psi+}$ versus $\rho(E) \bar{J}$ and $\rho(E) \Delta J$ for $k x_{0}=n \pi$ when the electron is injected in the state $|\uparrow\rangle$ with the impurities prepared in the state $|\downarrow \downarrow\rangle . \rho(E) \Delta J$ is normalized to $\rho(E) \bar{J}$, reducing to the ratio $\Delta J / \bar{J}$.

In conclusion, the results presented in this paper show very good tolerance of the entanglement-dependent transmission effects occurring in a $1 \mathrm{D}$ wire with two spin$1 / 2$ impurities [3] with respect to unavoidable static disorder in the coupling constants of the impurities. Therefore, the experimental difficulty in realizing two perfectly identical magnetic impurities does not appear to be an obstacle for the observation of such phenomena.

Acknowledgements Helpful discussions with J.-M. Lourtioz and G. Fishman are gratefully acknowledged. The authors thank the support from CNR (Italy) and GRICES (Portugal). YO and VRV thank the support from Fundação para a Ciência e a Tecnologia (Portugal), namely through programs POCTI/POCI and projects POCI/MAT/55796/2004 QuantLog and POCTI-SFA-291, partially funded by FEDER (EU).

* Electronic address: ciccarello@difter.unipa.it

[1] M. A. Nielsen, and I. L. Chuang, Quantum Computation and Quantum Information, Cambridge Univ. Press, Cambridge, (2000)

[2] G. Burkhard, D. Loss, and E. V. Sukhorukov, Phys. Rev. B 61, R16303 (2000); P. Samuelsson, E. V. Sukhorukov and M. Büttiker, Phys. Rev. B 70, 115330 (2004); F. Taddei and R. Fazio, Phys. Rev. B 65, 075317 (2002).

[3] F. Ciccarello, G. M. Palma, M. Zarcone, Y. Omar and V. R. Vieira, New J. Phys. 8, 214 (2006); F. Ciccarello, G. M. Palma, M. Zarcone, Y. Omar and V. R. Vieira, J. Phys. A: Math. Theor. 40, 7993 (2007)

[4] A. Stern, Y. Aharonov, and Y. Imry, Phys. Rev. A 41, 3436 (1990); S. K. Joshi, D. Sahoo, and A. M. Jayannavar, Phys. Rev. B 64, 075320 (2001).

[5] A. T. Costa, Jr., S. Bose, and Y. Omar, Phys. Rev. Lett. 96, 230501 (2006). 